\title{
ICT4Life Open Source Libraries supporting Multimodal Analysis of different diseases
}

\author{
Thomas Theodoridis, Vassilis Solachidis, Nicholas Vretos, Petros Daras \\ Dario Dotti, Mirela Popa
}

Gustavo Hernández, Federico Álvarez

Alejandro González Patón, Angel Lopez

\begin{abstract}
The ICT4Life Open Source framework contains libraries for acquiring and processing data from different sensors, machine learning algorithms for activity recognition, as well as fusion methods of multiple modalities either at an early or at a late stage. The main purpose of the introduced system is to enable an easy customization of patients' monitoring using different types of sensors. Furthermore, by allowing an easy integration of new sensors or types of activities, the proposed subsystem supports the development of new solutions for different diseases, than the ones considered in the ICT 4 Life project.
\end{abstract}

\section{Introduction}

The scope of the ICT4Life project [1] is to offer new services for integrated care, facilitating and promoting an improved quality of life and autonomy at home for patients with Parkinson's and Alzheimer's and their caregivers. As many patients prefer living at home, their constant monitoring using different sensors contributes at detecting critical situations (e.g. falling down or leaving the house while being in a confused state of mind) and helps gathering statistics about their behavioural patterns and detecting deviations from their normal routine. The analysis of their activity patterns is fused with their medical information, supporting the early detection of any alterations of their health condition. The timely communication with their care-givers and medical professionals supports a better decision making process, 


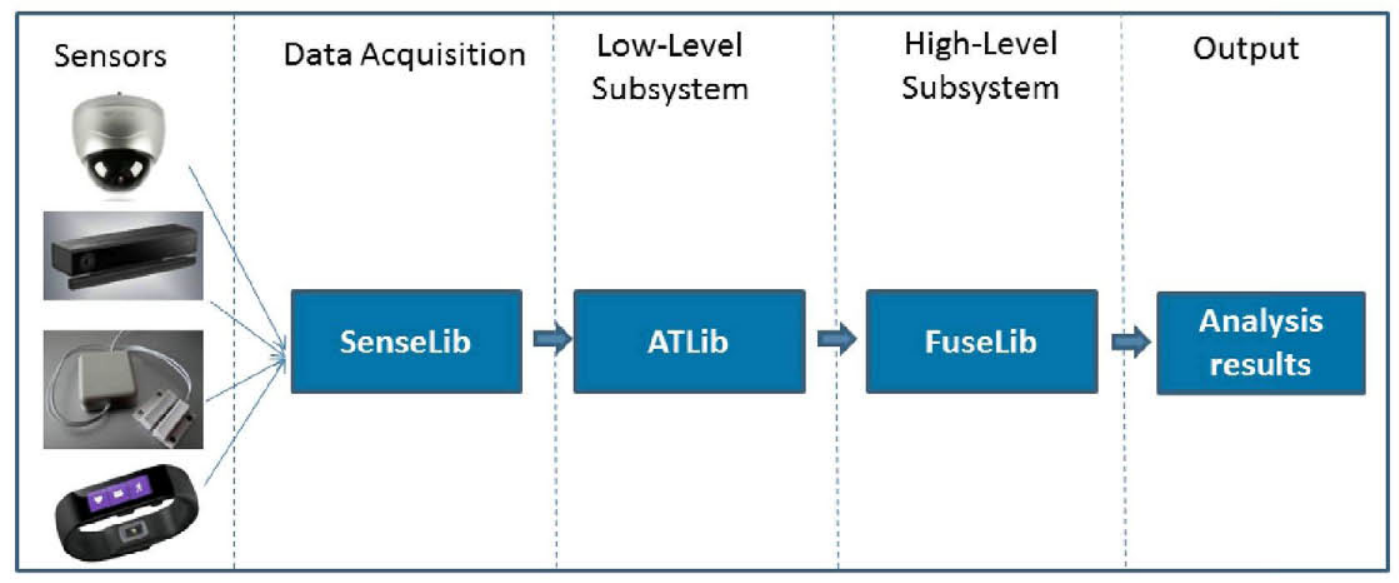

Figure 1: ICT4Life Open Source Libraries System Architecture.

leading to an improved and integrated care model. The presented approach is formed of several modules, which are made public for promoting research and fostering new markets. They form the ICT4Life Open Source Libraries, which are presented and described in this paper. Their main advantage is that they can be easily extended and integrated into new platforms, ensuring sustainability, a viable business solution as well as reusability of resources and knowledge. Furthermore, the proposed subsystem constitutes a cost-effective way of adapting the platform to the needs of potential clients. The Open Source Libraries subsystem is wellstructured and includes several libraries. The SenseLib library contains methods responsible for acquiring data from different sensors (e.g. cameras, depth sensors, ambient sensors and wearable devices) as well as methods for processing the acquired data, while also allowing integration of new sensors in the activity recognition process. The ATLib library offers several machine learning algorithms to be used in the activity recognition modules and allows new activities and patient behaviours to be trained. Finally, the FuseLib library contains different methods for customizing the way in which modalities can be employed in the fusion engine. All provided libraries are open-source, easy to customize and to extend to new functionalities, according to the user requirements.

In this paper, we present a solution for building a data-analysis framework useful in the health-care domain, which can be easily adapted to new use-cases, such as a different disease than the ones already considered in the ICT4Life project. As many elderly have more than one disease, the proposed framework also provides a solution for dealing with co-morbidities, by analysing the relationships between them in the FuseLib library.

\section{Related Work}

In the last years, many ICT projects focused on the health-care domain, aiming at fostering research by providing open source libraries. One example is Motmot [4], an open-source toolkit for real-time video acquisition and analysis useful in neuroscience applications.

Another Open-Source Application useful for neuroscientists, offering processing and visualization of MEG/EEG data is Brainstorm [5], a toolbox implemented in Matlab. Furthermore, Zephyr Open [6] is an open source code project, which provides a framework for BlueTooth physiological sensors, enabling heart rate and blood pressure monitoring. 


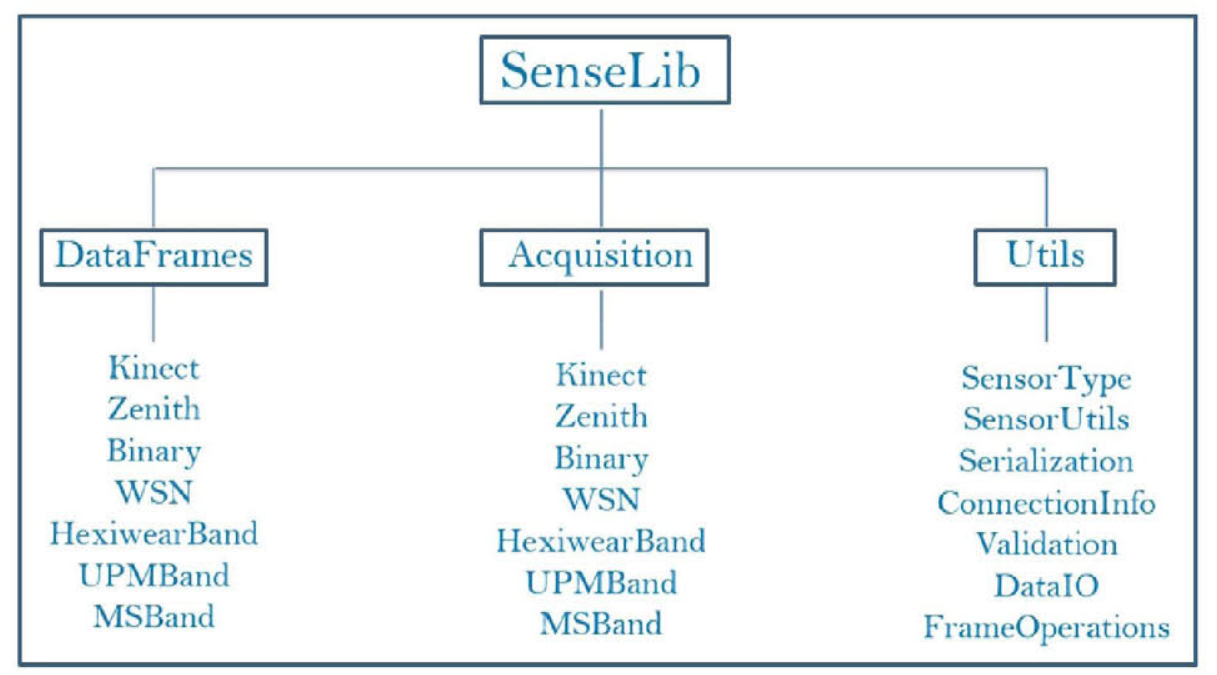

Figure 2: Architecture of the SenseLib library.

Each of the introduced open-source solutions are valuable, while they usually focus on one type of sensory data. This aspect is addressed by our proposed framework, by offering support for multiple types of sensors, data types and activity models.

\section{ICT4Life Open Source Libraries System Architecture}

The proposed open source subsystem is formed of several libraries, which can be used separately, but also together for developing a framework useful for analysing a different type of disease, than the ones considered in the ICT4Life project (e.g. Parkinson's and Alzheimer's). In Figure 1, the relation between the libraries is depicted, showing the pipeline from data acquisition using a set of sensors (e.g. cameras, depth sensors, wearable devices, binary sensors), feature extraction and classification for each defined modality and finally the fusion of the different modalities for obtaining a better decision, based on all available information. The description of each library is presented next, along with its internal architecture.

\subsection{SenseLib}

The Senselib library (available at https://github.com/tomastheod-ITI/SenseLib) contains methods responsible for acquiring and processing data from sensors. Examples of functionalities provided for video sensors are acquisition, compression, decompression and scaling of images, while for depth sensors, the bounding boxes and the filtered skeletons joints of a person are offered, contributing to activity recognition.

The library methods are organized in three main namespaces: the DataFrames, the Acquisition and the Utils namespaces, depicted in Figure 2. The first namespace contains the appropriate variables needed by each sensor for storing the data acquired from that device. The Acquisition namespace contains the methods needed to interact with each sensor, such as sensor initialization, checking its availability and starting the data acquisition process. Finally, the Utils namespace provides a plethora of different functionalities such as image compression, image scaling, video creation and database storage. 


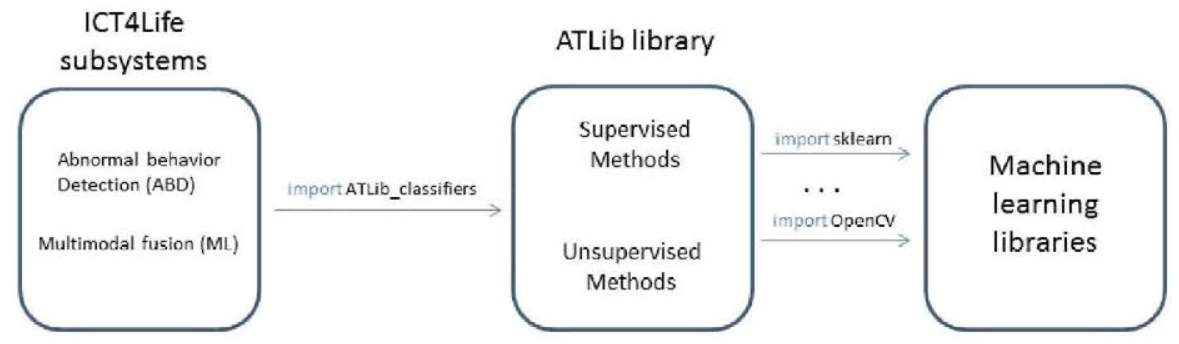

Figure 3: Architecture of the ATLib library.

\subsection{ATLib}

The ATLib library offers several machine learning algorithms to be used in the activity recognition modules of ICT4Life. The main advantages of the ATLib library consist of providing a common infrastructure for all the ICT4Life subsystems that use machine learning algorithms, simplifying and reducing the dependencies of the ICT4Life system on many different machine learning software modules and accelerating the usage of supervised and unsupervised learning methods across all system modules.

A wide range of supervised learning algorithms are available in the ATLib library, each with its strengths and weaknesses. There is no single learning algorithm that works best on all supervised learning problems. Therefore, one needs to take into consideration the characteristics of the input data, such as the range of features and their underlying distribution, in order to choose the most suitable method for solving a concrete domain problem. In ATLib we included the most used techniques from widely employed machine learning libraries like scikit-learn and $\mathrm{OpenCv}[2]$. The open source library can be downloaded from: https://github.com/dariodotti/ATLib. The architecture of the ATLib library is depicted in Figure 3, while its design facilitates training new activities or patient behaviours. The library was already employed at recognizing different activities, such as working patterns in an office or discriminating between confusion and normal behaviour patterns.

\subsection{FuseLib}

The FuseLib library offers different ways in which modalities can be employed in the fusion engine. There is a large set of options, depending on the reasoning type or the stage at which the fusion is performed. The main role of the library is to allow flexibility in customizing the way in which the different modalities can be combined for extracting a general conclusion regarding the level of the considered disease. The architecture of the FuseLib library is depicted in Figure 4 below.

Initially, different modalities are fed into the library, such as for example the patient medical profile, the social status and the user activity-related variables. The fusion mechanism can be based on either a deterministic or a probabilistic reasoning model. Usually, the probabilistic approach is preferred over the deterministic one, as is better at handling uncertainties and noisy data measurements. Next, the fusion algorithm can be applied either at the beginning or at the end of the processing pipeline, depending on the properties of each modality. The software implementation of the library is available at: https://github.com/mirelapopa/FuseLib. 


\section{FuseLib}

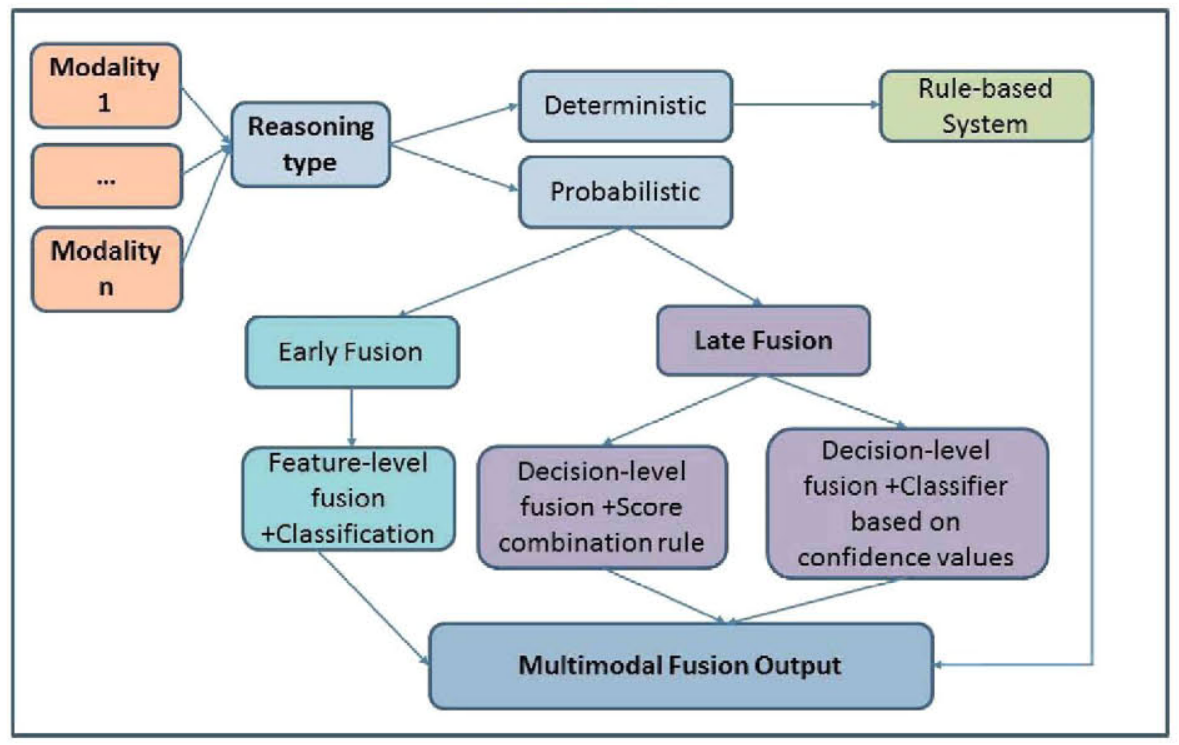

Figure 4: Architecture of the FuseLib library.

\section{Examples of potential applica- tions}

\subsection{SenseLib}

SenseLib can be extended in order to support acquiring data from new sensors. The minimum requirements for this is that a new frame for storing the data of the new sensor is added inside the DataFrames namespace and at least the common methods (OpenSensor, CloseSensor, UpdateConnectionParams, etc.) are implemented inside a new class in the Acquisition namespace. Providing additional functionalities for the new sensor, such as writing to the database, requires modifying the existing methods in the Utils namespace, such that the new sensor is taken into account. In the course of the ICT4Life project, the library has been already extended with new sensors, such as wearable devices (e.g. the UPM Band and the Hexiwear Band). For example, in the case of the Kinect sensor, the available methods enable the acquisition of the user trajectories inside the monitored space, while for the ambient sensors, the location and the temporal information, where and when the user interacted with specific objects are provided.

\subsection{ATLib}

Methods available in the ATlib library were successfully employed at discovering normal and abnormal behaviour patterns in an unsupervised manner [3]. The experiments performed on both indoor and outdoor datasets achieved a good overall accuracy, highlighting the possibility of applying the proposed methodology in different scenarios (e.g. a daily care centre or a patient's house) and for finding new behaviour patterns (e.g. elderly common activity patterns). For example, a potential application of ATlib consists of employing the user joint trajectories provided by SenseLib for 
training a movement evolution module, which can detect alterations of the psychical condition of the patient. The results of ATLib will be fed to the FuseLib, representing one of the analysed modalities.

\subsection{FuseLib}

The FuseLib library can be applied to other diseases, such as diabetes or cardiovascular diseases, as long as there are more modalities available, containing complementary information. The medical features provided in the case of diabetes will reflect the blood sugar level and the albumin creatinine ratio instead of the mobility indicators useful for Parkinson's disease, or the cognitive indicators in case of dementia. Furthermore, the user can interact with the different fusion approaches, check the accuracy results and select the most appropriate method for the investigated case. For example, the user can choose a certain type of fusion or test all available algorithms for finding the best performing one. The flexibility of the fusion library resides also in the possibility of customizing the weight of each modality in the fusion process, based on semantics or by discovering them in an automatic way during the training process, where the data statistical properties are investigated. For example, the fusion process can be useful at investigating the causes of the deviations in the patient behavioural patterns and raise an alert only in some cases (e.g. the deviations are observed over several days and they are not linked to a common disease or an external event). More specifically, if the patient is less active than usual, can be due to a cold, sleeping problems or due to apathy, case in which the medical information can provide additional details (e.g. the patient has a mild form of depression). Only in the last case, a notification should be sent to the caregiver, while the other cases can be treated in a more relaxed way, as the symptoms will probably disappear in a short period of time.

\section{Conclusions}

We described a set of open source libraries developed in the context of the ICT4Life project $^{1}$, useful at acquiring and processing sensory information, analyzing activity models and fusing several modalities for extracting meaningful information regarding the health condition of the monitored patient. The overall system architecture was introduced, showing the connections between the libraries. Next, we highlighted the potential applications of each library, such as incorporating new sensors, new activity models or scenarios, and even a new type of disease. In order to increase the reusability and the impact of the proposed framework, we plan as future work to test its efficiency in different set-ups, consisting of varying sets of sensors, activities and environments.

\section{References}

[1] ICT4Life: ICT Services for Life Improvement for the Elderly. http://ict4life.eu/, 2016-2018.

[2] G. Bradski. The OpenCV Library. Dr. Dobb's Journal of Software Tools, 2000.

[3] D. Dotti, M. Popa, and S. Asteriadis. Unsupervised discovery of normal and abnormal activity patterns in indoor and outdoor environments. In 12th International Conference on Computer Vision Theory and Applications (VISAPP), 2017.

[4] D. Straw, Andrew and H. Dickinson, Michael. Motmot, an open-source toolkit for realtime video acquisition and analysis. Source Code for Biology and Medicine, 4(1):5, 2009.

[5] F. Tadel, S. Baillet, J. C. Mosher, D. Pantazis, and R. M. Leahy. Brainstorm: A User-Friendly Application for MEG/EEG Analysis. Computational Intelligence and Neuroscience, 2011.

[6] B. Zdanivsky. Zephyr open. http://www. verticalchallenge.org/zephyropen/, 2017.

\footnotetext{
${ }^{1}$ This work has been funded by the European Union' Horizon 2020 Research and Innovation Programme under Grant Agreement $N^{\circ} 690090$ (ICT4Life project)
} 